\title{
Three Generations of FLNA-Associated Periventricular Nodular Heterotopia
}

\author{
Grace E. Eisenbiegler ${ }^{a}$ Stephen A. Brown ${ }^{a}$ b \\ aThe University of Vermont Larner College of Medicine, Burlington, VT, USA; bepartment of \\ Obstetrics and Gynecology, The University of Vermont Medical Center, Burlington, VT, USA
}

\section{Keywords}

FLNA · Periventricular nodular heterotopia - Congenital neurologic malformation · Case report · Fetal MRI

\begin{abstract}
We present a family with 3 generations of FLNA gene-associated periventricular nodular heterotopia (PVNH), with a unique presentation in a fetus with multiple neurologic malformations. Neurologic abnormalities were noted on routine fetal imaging for a 33-year-old G1P0 woman; absence of the corpus callosum and PVNH was confirmed on follow-up MRI. This prompted genetic evaluation, revealing a nonsense mutation in the FLNA gene. Familial genetic analysis and neuroimaging revealed the same variant and MRI evidence of PVNH in the fetus's asymptomatic mother, and maternal grandmother, who had a long history of seizure disorder. Such phenotypic variability within a single family demonstrates the spectrum of $\mathrm{PVNH}$ and the importance of genetic counseling for patients with PVNH. This case also adds to existing literature on the rare but not unique presentation of FLNA-associated fetal malformations.
\end{abstract}

(C) 2021 The Author(s).

Published by S. Karger AG, Basel

\section{Introduction}

Periventricular nodular heterotopia (PVNH) is a congenital disorder of neuronal migration characterized by nodules of ectopic neurons situated adjacent to the walls of the lateral cerebral ventricles. Several etiologies for PVNH have been described, and a common cause of this condition is mutation of the FLNA gene, which is found in about $50 \%$ of female patients with bilateral anterior PVNH [1]. FLNA encodes Filamin A, an actin-binding protein that plays 
key roles in cytoskeletal structural development, cell migration, and vascular development and stability [2]. Most patients with FLNA mutations present with seizures, however, other complications include cardiovascular, intestinal, and musculoskeletal anomalies. Associated neurologic malformations include thin corpus callosum in $48 \%$ of patients with callosal agenesis in $12 \%$, as well as cerebellar dysgenesis in $54 \%$ of patients [3]. We present a threegeneration family with PVNH due to a novel FLNA variant with highly variable clinical presentations, demonstrating the pleiotropy associated with mutations at this locus. This case reports demonstrates a unique spectrum of presentations, including a rarer presentation in a fetus.

\section{Case}

A 33-year-old G1P0 presented for fetal ultrasound evaluation at 21 weeks and 6 days gestation due to concern for cerebral ventriculomegaly on a prior scan. Although the ventricles were not enlarged on ultrasound, the cavum septum pellucidum and corpus callosum were not visualized. Additionally, a $12 \mathrm{~mm} \times 5 \mathrm{~mm}$ interhemispheric cystic area and hypoplasia of the cerebellar vermis were noted. Subsequent fetal MRI shown in Figure 1 confirmed absence of the corpus callosum and demonstrated PVNH. After counseling about the likelihood of a poor neurologic outcome, the patient elected pregnancy termination.

Termination was completed at 23 weeks, and tissue was sent for chromosome microarray and a comprehensive brain malformation gene sequencing panel. The microarray was normal 46,XX, while gene sequencing demonstrated a novel heterozygous variant in FLNA: c.5977delC, which predicts a frameshift and premature stop (p.Arg1993GlyfsX23) in the Filamin A protein.

We predicted that this mutation occurred de novo in the fetus. However, considering the wide variability in FLNA-associated phenotype, parental testing was initiated and subsequently demonstrated the same variant in the mother. Because of the known association of FLNA mutations and cerebral vascular malformations, an MR-angiogram was performed. This demonstrated normal cerebral blood vessels as well as extensive PVNH, shown in Figure 2. This unexpected finding changed reproductive counseling significantly, due to the $50 \%$ chance of transmission of the variant and its unpredictable phenotypic consequences.

During discussion, the patient noted that her mother had a long-standing seizure disorder. Because of this history, a report from an MRI performed in 2009 was obtained. Although images
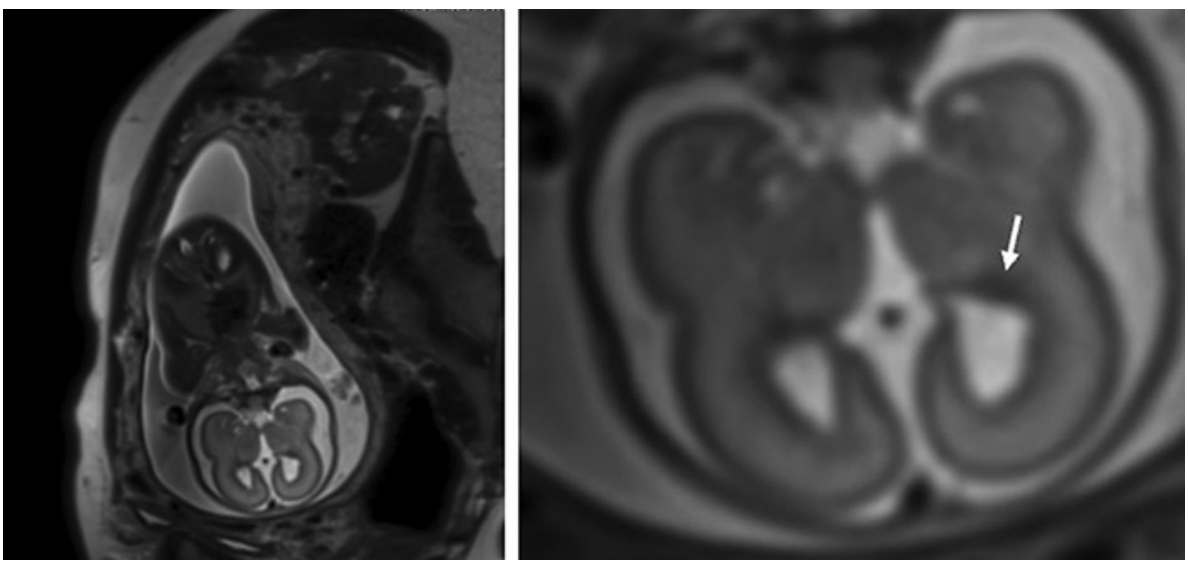

Fig. 1. MRI demonstrating the fetus within the mother's uterus. The coronal image of the fetal brain demonstrates agenesis of the corpus callosum as well as PVNH (marked with the arrow within inset). PVNH, periventricular nodular heterotopia.

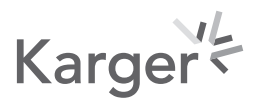


Fig. 2. MRI image of the brain of the mother, demonstrating extensive PVNH. PVNH, periventricular nodular heterotopia.

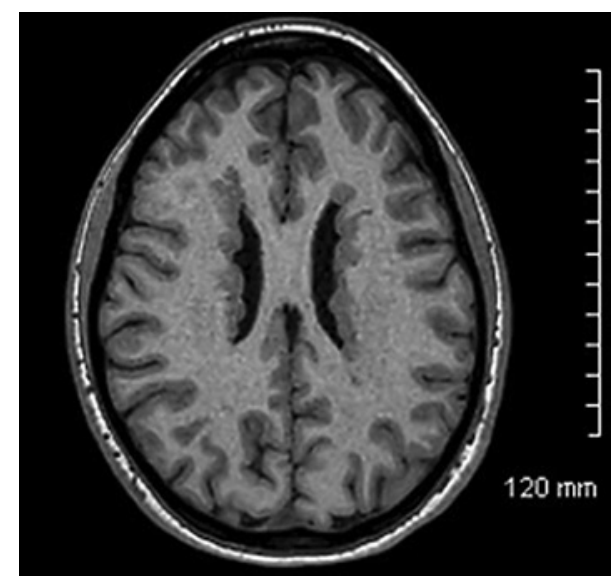

Fig. 3. Pedigree with filled circles indicating the 3 family members with the FLNA variant.

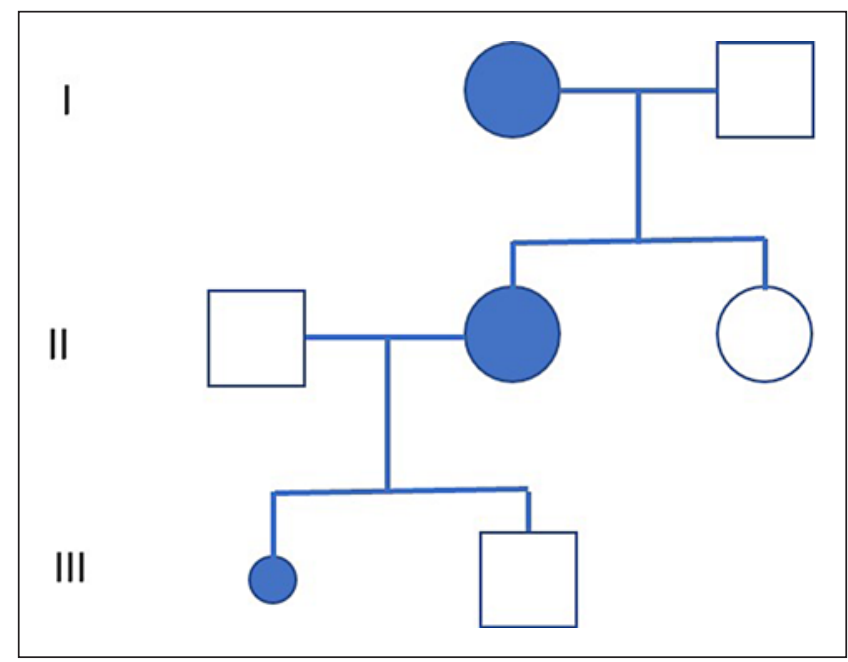

were not available, the report described extensive PVNH. This finding prompted genetic testing, which confirmed the presence of the FLNA variant in the patient's mother as well. Family pedigree can be seen in Figure 3.

\section{Discussion}

We report a 3-generation family presenting with FLNA-associated PVNH as well as a variety of other features that have been associated with FLNA mutations. Although the family we report is striking, it is not entirely unique. Chen et al. [4] reported a patient who had an incidental finding of PVNH when she underwent MRI due to headache. She subsequently had a daughter who developed seizures as a child and was found to also have PVNH. Analysis of FLNA demonstrated a predicted loss-of-function mutation in both mother and daughter. Interestingly, a subsequent pregnancy in this patient ended with growth restriction, oligohydramnios, and demise of a female fetus at 24 weeks, raising the possibility that FLNA mutation might lead to fetal demise, perhaps due to extremely skewed X-chromosome inactivation. Genetic testing of the fetus identified the same FLNA mutation, but, unfortunately, studies of $\mathrm{X}$-chromosome inactivation skewing were not performed [4]. Another report Stoecklein et al. 
[5] described 2 pregnancies in the same patient, each with a fetus with bilateral PVNH and megacisterna magna, identified with fetal MRI. Both fetuses had inherited an FLNA mutation from the clinically normal mother, prompting neuroimaging of the mother, which revealed PVNH [5].

The present case demonstrates the high degree of phenotypic variability in individuals with FLNA loss-of-function variants. The discovery of the familial mutation changed genetic counseling for this family, as the patient was advised of the $50 \%$ chance of transmission of the variant in a subsequent pregnancy with unknowable phenotypic presentation of the fetus. This family, as well similar cases in the literature, suggest that individuals with PVNH who are looking to conceive should be offered genetic evaluation prior to pregnancy, to allow better awareness, and therefore assessment, of possible heritable mutations that would lead to deleterious neurologic outcomes for the fetus. Additionally, asymptomatic parents of children with PVNH should be offered screening for PVNH, due to the strong heritability of the syndrome and the possibility of subclinical disease in the parents. This case also demonstrates the variations in severity and prognosis within the spectrum of PVNH, broadening the documented presentation of this neurologic disease. The limitations of this report include inability to access neuroimaging performed outside our institution for one of the patient's described and the determination that the genetic variant in question is a "variant of unknown significance," though it was highly likely to be pathogenic based on its predicted loss of function due to protein truncation. Nonetheless, because it was not present in ClinVar or other databases of known pathogenic variants, it is classified as a variant of unknown significance.

\section{Statement of Ethics}

The lead author affirms that this manuscript is an honest, accurate, and transparent account of the study being reported that no important aspects of the study have been omitted. Ethics approval was not required for this study, as there was no intervention performed and all personally identifiable information has been withheld. Additionally, disclosure of the de-identified information in this case report does not risk emotional, physical, or financial harm to the subjects described. Informed written consent for publication of this case report and accompanying images was obtained from the patients described in this case report. Signed consent forms were obtained and will be filed with our records.

\section{Conflict of Interest Statement}

The authors have no financial, personal, or professional disclosures relevant to the manuscript. The authors have no financial interest or benefit that has arisen from the direct application of this research.

\section{Funding Sources}

This study received no funding. 


\section{Author Contributions}

Dr. Stephen Brown contributed to the conception and design of this report. Grace Eisenbiegler drafted the work. Both authors revised this work and contributed to the discussion

\section{References}

1 Parrini E, Ramazzotti A, Dobyns WB, Mei D, Moro F, Veggiotti P, et al. Periventricular heterotopia: phenotypic heterogeneity and correlation with filamin A mutations. Brain. 2006;129(7):1892-906.

2 Lange M, Kasper B, Bohring A, Rutsch F, Kluger G, Hoffjan S, et al. 47 patients with FLNA associated periventricular nodular heterotopia. Orphanet J Rare Dis. 2015;10:134.

3 Pisano T, Barkovich AJ, Leventer RJ, Squier W, Scheffer IE, Parrini E, et al. Peritrigonal and temporo-occipital heterotopia with corpus callosum and cerebellar dysgenesis. Neurology. 2012;79:1244-51.

4 Chen C-P, Chern S-R, Chiu N-C, Liu YP, Chen YN, Chen SW, et al. Detection of a novel c.7106_7110delinsT heterozygous mutation in the FLNA gene in an asymptomatic mother with periventricular nodular heterotopia during prenatal genetic counseling. Taiwan J Obstet Gynecol. 2016;55(6):897-9.

5 Stoecklein S, Haberler C, Gruber G, Diogo M, Ulm B, Laccone FA, et al. Bilateral periventricular nodular heterotopia detected on fetal and maternal MRI attributable to novel filamen A gene mutation. Ultrasound Obstet Gynecol. 2018;52:677-81. 